\title{
QCD Spin Physics: Partonic Spin Structure of the Nucleon*
}

\author{
D. de Florian, ${ }^{1}$ R. Sassot, ${ }^{1}$ M. Stratmann, ${ }^{2}$ W. Vogelsang ${ }^{3}$ \\ ${ }^{1}$ Departamento de Fisica, Universidad de Buenos Aires, \\ Ciudad Universitaria, Pabellon 1 (1428) Buenos Aires, Argentina \\ ${ }^{2}$ Physics Department, Brookhaven National Laboratory, \\ Upton, NY 11973, U.S.A. \\ ${ }^{3}$ Institute for Theoretical Physics, Universität Tübingen, \\ Auf der Morgenstelle 14, D-72076 Tübingen, Germany
}

August 17, 2018

\begin{abstract}
We discuss some recent developments concerning the nucleon's helicity parton distribution functions: New preliminary data from jet production at RHIC suggest for the first time a non-vanishing polarization of gluons in the nucleon. SIDIS measurements at COMPASS provide better constraints on the strange and light sea quark helicity distributions. Single-longitudinal spin asymmetries in $W$-boson production have been observed at RHIC and will ultimately give new insights into the light quark and anti-quark helicity structure of the nucleon.
\end{abstract}

\section{Introduction}

QCD spin physics has been driven by the hugely successful experimental program of polarized deeplyinelastic lepton-nucleon scattering (DIS) [1. One of the most important results has been the finding that the quark and anti-quark spins (summed over all flavors) provide only about a quarter of the nucleon's spin, $\Delta \Sigma \approx 0.25$ in the proton helicity sum rule [2]:

$$
\frac{1}{2}=\frac{1}{2} \Delta \Sigma+\Delta G+L_{q}+L_{g}
$$

This result implies that sizable contributions to the nucleon spin should come from the gluon spin contribution $\Delta G$, or from orbital angular momenta $L_{q, g}$ of partons. To determine the other contributions to the nucleon spin has become a key focus of the field. In the present article, we describe some of the recent developments of the field. We focus on current efforts to determine the helicity parton distributions of the nucleon and on the latest experimental results.

The helicity structure of the nucleon is foremost described by its twist-two helicity parton distribution functions,

$$
\Delta f\left(x, Q^{2}\right) \equiv f^{+}\left(x, Q^{2}\right)-f^{-}\left(x, Q^{2}\right) \quad(f=u, d, s, \bar{u}, \bar{d}, \bar{s}, g),
$$

${ }^{*}$ Talk presented by W. Vogelsang 
$f^{+}\left(f^{-}\right)$denoting the number density of partons with the same (opposite) helicity as the nucleon's, as a function of momentum fraction $x$ and scale $Q$. QCD predicts the $Q^{2}$-dependence of the densities through the spin-dependent Dokshitzer-Gribov-Lipatov-Altarelli-Parisi (DGLAP) evolution equations [3]:

$$
\frac{d}{d \ln Q^{2}}\left(\begin{array}{c}
\Delta q \\
\Delta g
\end{array}\right)\left(x, Q^{2}\right)=\left(\begin{array}{cc}
\Delta P_{q q}\left(\alpha_{s}, x\right) & \Delta P_{q g}\left(\alpha_{s}, x\right) \\
\Delta P_{g q}\left(\alpha_{s}, x\right) & \Delta P_{g g}\left(\alpha_{s}, x\right)
\end{array}\right) \otimes\left(\begin{array}{c}
\Delta q \\
\Delta g
\end{array}\right)\left(x, Q^{2}\right)
$$

where $\otimes$ denotes a convolution, and the splitting functions $\Delta P_{i j}$ are evaluated in QCD perturbation theory [3, 4, 5].

The contributions $\Delta \Sigma\left(Q^{2}\right)$ and $\Delta G\left(Q^{2}\right)$ in the helicity sum rule $(1)$ are given by

$$
\begin{aligned}
& \Delta \Sigma\left(Q^{2}\right)=\int_{0}^{1}(\Delta u+\Delta \bar{u}+\Delta d+\Delta \bar{d}+\Delta s+\Delta \bar{s})\left(x, Q^{2}\right) d x \equiv \Delta \Sigma_{u}+\Delta \Sigma_{d}+\Delta \Sigma_{s}, \\
& \Delta G\left(Q^{2}\right)=\int_{0}^{1} \Delta g\left(x, Q^{2}\right) d x .
\end{aligned}
$$

$\Delta \Sigma$ is independent of $Q^{2}$ at the lowest order. The distributions have a proper field-theoretic definition. For example, in case of $\Delta g$ it is given by [6]

$$
\Delta g\left(x, Q^{2}\right)=\left.\frac{i}{4 \pi x P^{+}} \int d \lambda \mathrm{e}^{i \lambda x P^{+}}\left\langle P, S\left|G^{+\nu}(0) \tilde{G}_{\nu}^{+}(\lambda n)\right| P, S\right\rangle\right|_{Q^{2}}
$$

written in $A^{+}=0$ gauge. Here, $G^{\mu \nu}$ is the QCD field strength tensor, and $\tilde{G}^{\mu \nu}$ its dual. The integral of $\Delta g\left(x, Q^{2}\right)$ over all momentum fractions $x$ becomes a local operator only in $A^{+}=0$ gauge and then coincides with $\Delta G\left(Q^{2}\right)[2]$.

The helicity parton distributions may be probed in spin asymmetries for reactions at large momentum transfer. The probes used so far are inclusive and semi-inclusive deep-inelastic lepton scattering (DIS and SIDIS, respectively), and $p p$ scattering at large transverse momentum, see Fig. 1. Polarized DIS and SIDIS experiments have been carried out at SLAC, CERN, DESY and the Jefferson Laboratory [1] and mostly constrain the quark and anti-quark helicity distributions. RHIC at BNL [7, 8] is the first polarized proton-proton collider, operating at $\sqrt{s}=200$ and $500 \mathrm{GeV}$. The measurement of gluon polarization in the proton is a major focus and strength of RHIC.

The basic theoretical concept that underlies much of spin physics is the factorization theorem. It states that large momentum-transfer reactions may be factorized into long-distance pieces that contain

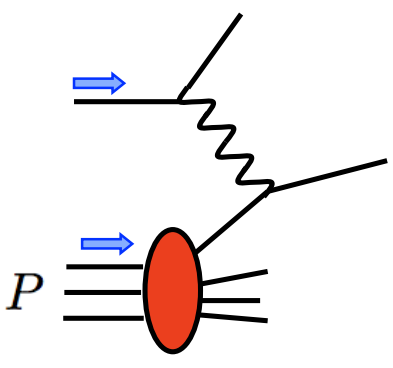

DIS

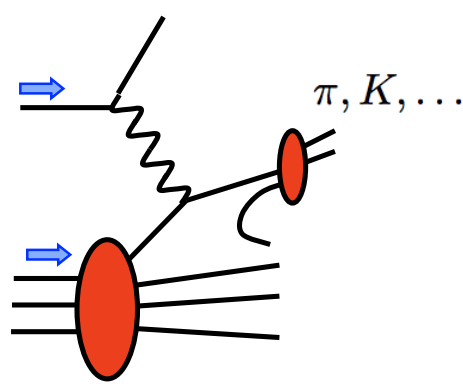

SIDIS

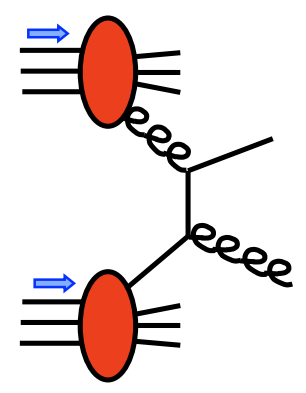

pp (RHIC)

Figure 1: Parton-model Feynman diagrams for the processes constraining nucleon helicity structure. 
the desired information on the spin structure of the nucleon in terms of its universal parton densities, and parts that are short-distance and describe the hard interactions of the partons. The latter can be evaluated using perturbation theory, thanks to the asymptotic freedom of QCD. As an example, we consider the reaction $p p \rightarrow \pi X$, where the pion is produced at high transverse momentum $p_{T}$, ensuring large momentum transfer. The statement of the factorization theorem [9] is then:

$$
d \Delta \sigma=\sum_{a, b, c} \Delta f_{a} \otimes \Delta f_{b} \otimes d \Delta \hat{\sigma}_{a b}^{c} \otimes D_{c}^{\pi}
$$

for the polarized cross section, where $\otimes$ denotes a convolution. The $D_{c}^{\pi}$ are the pion fragmentation functions. The sum in Eq. (7) is over all contributing partonic channels $a+b \rightarrow c+X$, with $d \Delta \hat{\sigma}_{a b}^{c}$ the associated spin-dependent partonic cross section. Factorization is valid up to corrections that are suppressed as inverse powers of the hard scale. In general, a leading-order estimate of (7) merely captures the main features, but does not usually provide a quantitative understanding. Only with knowledge of the next-to-leading order (NLO) QCD corrections to the $d \Delta \hat{\sigma}_{a b}^{c}$ can one reliably extract information on the parton distribution functions from the reaction. By now, NLO corrections are available for most of the processes relevant in polarized high-energy scattering [10].

Independent information on the nucleon's helicity distributions may be obtained by using SU(2) and $\mathrm{SU}(3)$ flavor symmetries. The integrals of the flavor non-singlet combinations turn out to be proportional to the nucleon matrix elements of the quark non-singlet axial currents, $\left\langle P, S\left|\bar{q} \gamma^{\mu} \gamma^{5} \lambda_{i} q\right| P, S\right\rangle$. Such currents typically occur in weak interactions, and one may relate the matrix elements to the $\beta$-decay parameters $F, D$ of the baryon octet. One finds

$$
\begin{aligned}
& \Delta \Sigma_{u}-\Delta \Sigma_{d}=F+D=1.267 \\
& \Delta \Sigma_{u}+\Delta \Sigma_{d}-2 \Delta \Sigma_{s}=3 F-D \approx 0.58 .
\end{aligned}
$$

If valid, the second relation when combined with Eq. (4) gives that $\Delta \Sigma=0.58+3 \Delta \Sigma_{s}$, so that a small quark spin contribution to the proton spin implies a large negative strange quark contribution. Fairly significant violations of SU(3) symmetry have been predicted based on heavy baryon chiral perturbation theory [11. Lattice investigations of this issue have begun but are not yet conclusive [12].

\section{Nucleon helicity structure: status 2009}

In recent publications [13, we have presented the first next-to-leading order (NLO) "global" QCD analysis of the nucleon's helicity distribution from DIS, semi-inclusive DIS (SIDIS), and $p p$ scattering at RHIC. We have used a Mellin moment method for the analysis. Our results are shown in Fig. 2, along with estimates of their uncertainties. The shaded bands in Fig. 2 show the distributions that are allowed if one permits an overall increase of $\Delta \chi^{2}=1$ (green) or $\Delta \chi^{2} / \chi^{2}=2 \%$ (yellow). As one can see, the "total" $\Delta u+\Delta \bar{u}$ and $\Delta d+\Delta \bar{d}$ helicity distributions are very well constrained. This is expected since these distributions are primarily determined by the large body of inclusive DIS data. Our results agree well with the distributions obtained in previous and other recent analyses [14, 15, 16, 17, 18] which considered only the lepton scattering data.

The sea anti-quark distributions still carry rather large uncertainties, even though they are better constrained now than in previous analyses, thanks to the advent of more precise SIDIS data and of a new set of fragmentation functions [19] that describes the observables well in the unpolarized case. We find that the sea appears not to be $\mathrm{SU}(2)$-flavor symmetric: the $\Delta \bar{u}$ distribution is mainly positive, while the $\Delta \bar{d}$ anti-quarks carry opposite polarization. This pattern has been predicted at least qualitatively by a number of models [14, 20]. Already based on the Pauli principle one would expect that if valence- $u$

quarks primarily spin along the proton spin direction, $u \bar{u}$ pairs in the sea will tend to have the $u$ quark 

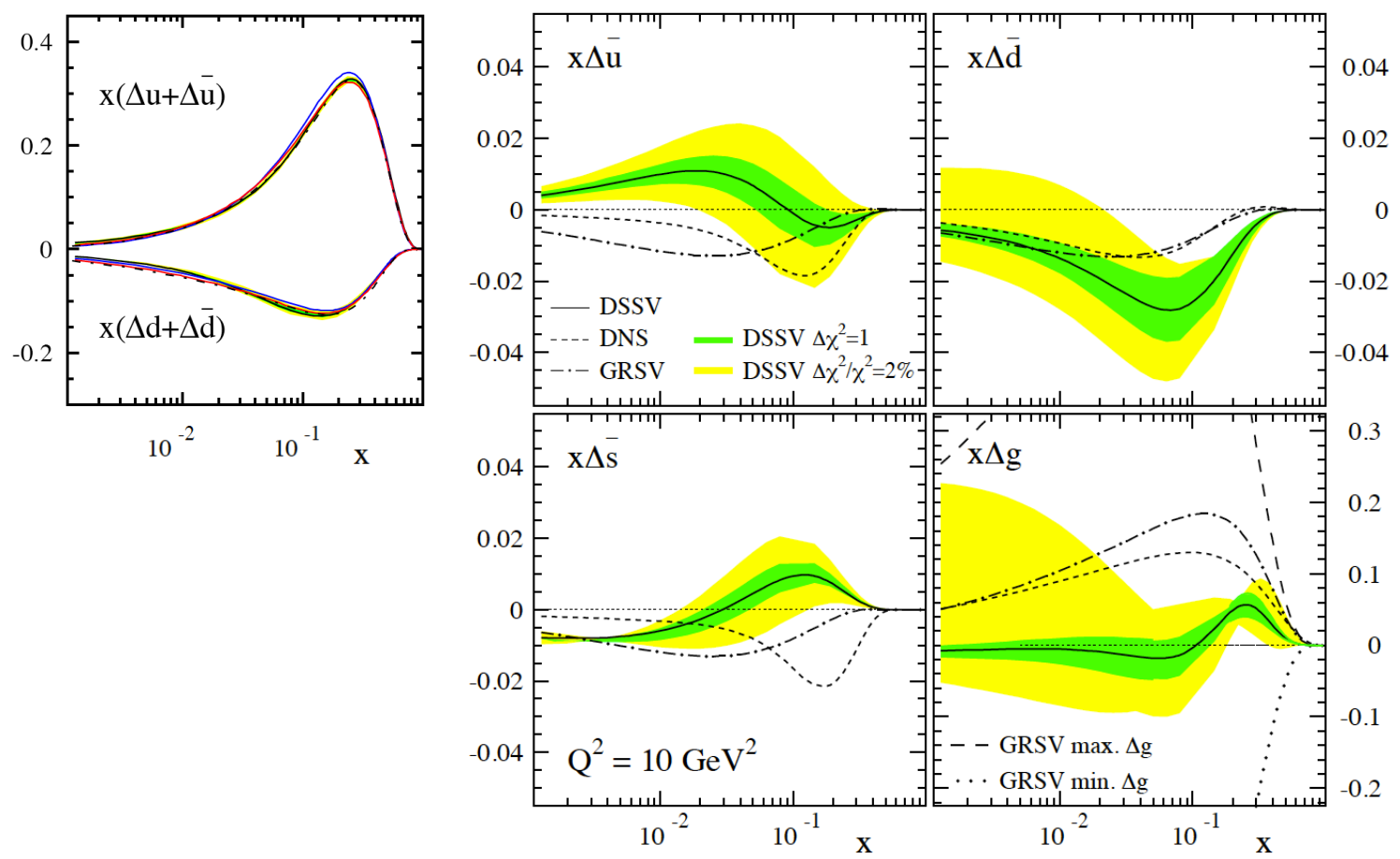

Figure 2: Present status of the nucleon's NLO helicity distributions according to the global analysis of Ref. [13. The solid center lines show the best-fit result. The shaded bands provide uncertainty estimates, using a criterion of $\Delta \chi^{2}=1$ (inner bands) or $\Delta \chi^{2} / \chi^{2}=2 \%$ (outer bands) as allowed tolerance on the $\chi^{2}$ value of the fit. Also shown are results from earlier analyses [14, 15] of nucleon spin structure from lepton scattering data alone.

polarized opposite to the proton. Hence, if such pairs are in a spin singlet, one expects $\Delta \bar{u}>0$ and, by the same reasoning, $\Delta \bar{d}<0$. We note that the uncertainties in SIDIS are still quite large, and it is in particular difficult to quantify the systematic uncertainty of the results related to the fragmentation mechanism at the relatively modest energies available.

The strange sea quark density shows a sign change. At moderately large $x \sim 0.1$, it is constrained by the SIDIS data, which prefer a positive $\Delta s$. On the other hand, the inclusive DIS data combined with the constraints from baryon $\beta$-decays demand a negative integral of $\Delta s$. As a consequence, $\Delta s$ obtains its negative integral purely from the contribution from low- $x$. Interestingly, there are initial lattice determinations of the integral $\Delta \Sigma_{s}$ [12], which point to small values. It is clearly important to understand the strange contribution to nucleon spin structure better.

Constraints on the spin-dependent gluon distribution $\Delta g$ predominantly come from RHIC. As can be seen from Fig. 2, the gluon distribution turns out to be small in the region of momentum fraction, $0.05 \lesssim x \lesssim 0.2$, accessible at RHIC, quite possibly having a node. At $Q^{2}=10 \mathrm{GeV}^{2}$, the integral over the mostly probed $x$-region is found to be almost zero, $\int_{0.05}^{0.2} d x \Delta g(x)=0.005 \pm 0.06$, where the error is obtained for a variation of $\chi^{2}$ by one unit. Thus, on the basis of [13], there are no indications of a sizable contribution of gluon spins to the proton spin. We also note that a way to access $\Delta g$ in lepton-nucleon scattering at HERMES and COMPASS is to measure final states that select the photon-gluon fusion process, heavy-flavor production and high- $p_{T}$ hadron or hadron-pair production [21, 22]. These data were not included in the analysis [13], mostly because of the fact that success of the perturbative-QCD hard-scattering description had not been established for these observables in the kinematic regime of 


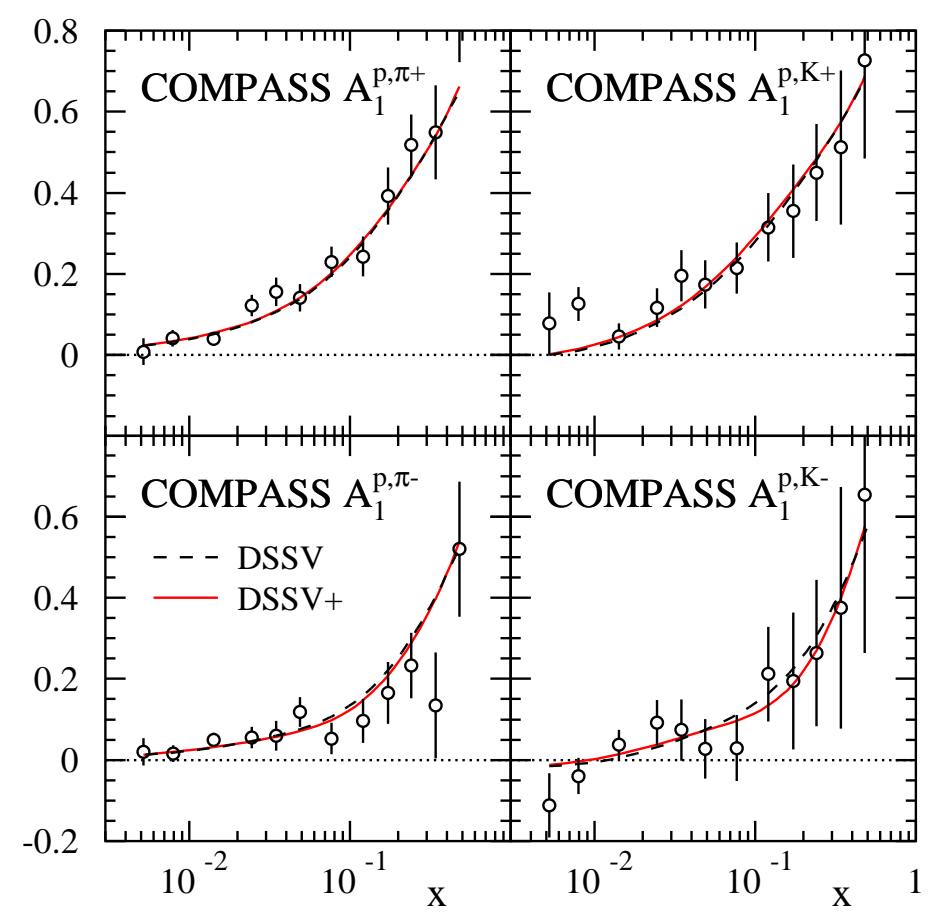

Figure 3: COMPASS results [27] for SIDIS spin asymmetries on a proton target, compared to DSSV [13] and DSSV+ fits [29].

interest here. At least for single-inclusive high- $p_{T}$ hadrons in $\gamma p \rightarrow h^{ \pm} X$ it has now been found that QCD hard-scattering does appear to be applicable in the COMPASS kinematic regime [23, 24].

\section{Recent developments}

Interesting new developments have taken place following the original DSSV analysis, mostly related to the advent of new data. We will summarize these in the following.

\subsection{Recent DIS and SIDIS data}

Recently, the COMPASS collaboration has published new DIS [25] and SIDIS [26, 27] data. The latter extend the coverage in $x$ down to about $x \simeq 5 \times 10^{-3}$, almost an order of magnitude lower than the kinematic reach of the HERMES data [28] used in the DSSV global analysis of 2008 [13]. For the first time, the new results comprise measurements of identified pions and kaons taken with a longitudinally polarized proton target. Clearly, these data can have a significant impact on fits of helicity PDFs and estimates of their uncertainties. In particular, the new kaon data are expected to serve as an important check of the validity of the strangeness density obtained in the DSSV analysis discussed above, which instead of favoring a negative polarization as in most fits based exclusively on DIS data, prefers a vanishing or perhaps even slightly positive $\Delta s$ in the measured range of $x$.

Figure 3 shows a detailed comparison [29] between the new proton SIDIS spin asymmetries from COMPASS [26, 27] and the original DSSV fit (dashed lines). Also shown is the result of a re-analysis at NLO [29] accuracy (denoted as "DSSV+") based on the updated data set. The differences between the DSSV and the DSSV + fits are hard to notice, both for identified pions and kaons. The total $\chi^{2}$ 

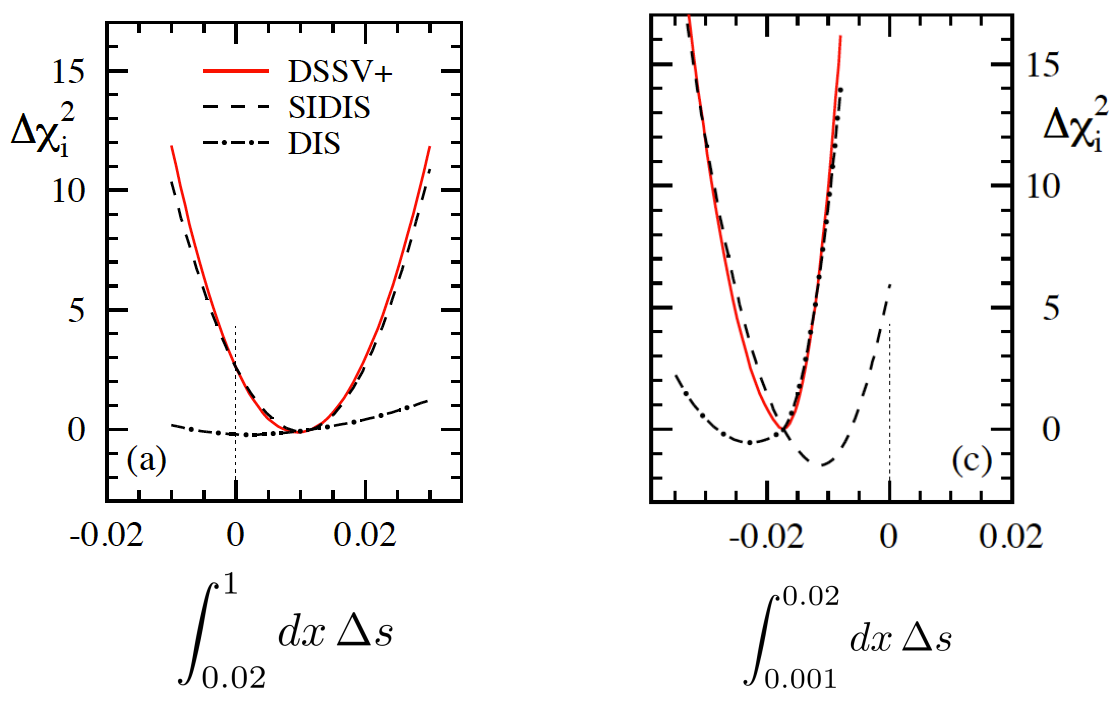

Figure 4: $\chi^{2}$ profiles for the truncated first moment of $\Delta s$ in two different $x$ intervals, $0.02 \leq x \leq 1$ (left) and $0.001 \leq x \leq 0.02$ (right).

of the fit drops only by a few units upon refitting, which is not really a significant improvement for a PDF analysis in view of non-Gaussian theoretical uncertainties. The change in $\chi^{2}$ is also well within the maximum $\Delta \chi^{2} / \chi^{2}=2 \%$ criterion adopted in the original DSSV global analysis [13]. Overall, upon refitting, one finds a trend towards smaller net polarization for $\Delta \bar{u}$ and $\Delta \bar{d}$ in the range $0.001 \leq x \leq 1$ than in DSSV.

As we saw earlier, the original DSSV fit [13] found an interesting feature for the strangeness helicity distribution: $\Delta s$ was found to be small and slightly positive at medium-to-large $x$, but has a significantly negative first moment in accordance with expectations based on $\mathrm{SU}(3)$ symmetry and fits to DIS data only. To investigate this issue further, we present in Fig. 4 the $\chi^{2}$ profiles for two different intervals in $x$ : $0.02 \leq x \leq 1$ (left) and $0.001 \leq x \leq 0.02$ (right). The profiles in Fig. 4 clearly show that the result for $\Delta s$ for $0.001 \leq x \leq 0.02$ is a compromise between DIS and SIDIS data, the latter favoring less negative values. Interestingly though, the new COMPASS SIDIS data, which extend towards the smallest $x$ values so far, actually show some preference for a slightly negative value for $\Delta s$ as well. For $0.02 \leq x \leq 1$ everything is determined by SIDIS data, and all sets consistently ask for a small, slightly positive strange quark polarization. There is no hint of a tension with DIS data here as they do not provide a useful constraint at medium-to-large $x$. We note that at low $x$ most SIDIS sets in the original DSSV fit give indifferent results. We also mention that in the range $x>0.001$ the hyperon decay constants, the so-called $F$ and $D$ values, do not play a significant role in constraining $\Delta s(x)$. To quantify possible $\mathrm{SU}(3)$ breaking effects one needs to probe $\Delta s(x)$ at even smaller values of $x$, for instance in SIDIS at a future EIC [30. We finally note that the HERMES and COMPASS data are consistent in the region of overlap, $0.02 \leq x \leq 1$.

Clearly, all current extractions of $\Delta s$ from SIDIS data suffer from a significant dependence on kaon FFs, see, e.g., Refs. [26, 27], and better determinations of $D^{K}(z)$ are highly desirable. Contrary to other fits of FFs [32], only the DSS sets [19] provide a satisfactory description of pion and kaon multiplicities in the same kinematic range where we have polarized SIDIS data. 


\subsection{W bosons at RHIC}

We have seen in the previous section that the SIDIS data provide some insights into the flavor structure of the polarized sea distributions of the nucleon, albeit with still fairly large uncertainties. Complementary and clean information on $\Delta u, \Delta \bar{u}, \Delta d$, and $\Delta \bar{d}$ will come from $p p \rightarrow W^{ \pm} X$ at RHIC, where one exploits the maximally parity-violating couplings of the produced $W$ bosons to left-handed quarks and right-handed anti-quarks [7, 33]. These give rise to a single-longitudinal spin asymmetry,

$$
A_{L} \equiv \frac{\sigma^{+}-\sigma^{-}}{\sigma^{+}+\sigma^{-}}
$$

for the processes $\overrightarrow{p p} \rightarrow \ell^{ \pm} X$, where the arrow denotes a longitudinally polarized proton and $\ell=e$ or $\mu$ is the charged decay lepton. The high scale set by the $W$ boson mass makes it possible to extract quark and anti-quark polarizations from inclusive lepton single-spin asymmetries in $W$ boson production with minimal theoretical uncertainties, as higher order and sub-leading terms in the perturbative QCD expansion are suppressed [34, 35, 36, 37].

For $W^{-}$production, neglecting all partonic processes but the dominant $\bar{u} d \rightarrow W^{-}$one, the spindependent cross section in the numerator of the asymmetry is found to be proportional to the combination

$$
\Delta \sigma \propto \Delta \bar{u}\left(x_{1}\right) \otimes d\left(x_{2}\right)(1-\cos \theta)^{2}-\Delta d\left(x_{1}\right) \otimes \bar{u}\left(x_{2}\right)(1+\cos \theta)^{2},
$$

where $\theta$ is the polar angle of the electron in the partonic c.m.s., with $\theta=0$ in the forward direction of the polarized parton. At large negative pseudorapidity $\eta_{\text {lept }}$ of the charged lepton, one has $x_{2} \gg x_{1}$ and $\theta \gg \pi / 2$. In this case, the first term in Eq. 10 strongly dominates, since the combination of parton distributions, $\Delta \bar{u}\left(x_{1}\right) d\left(x_{2}\right)$, and the angular factor, $(1-\cos \theta)^{2}$, each dominate over their counterpart in the second term. Since the denominator of $A_{L}$ is proportional to $\bar{u}\left(x_{1}\right) \otimes d\left(x_{2}\right)(1-\cos \theta)^{2}+d\left(x_{1}\right) \otimes$ $\bar{u}\left(x_{2}\right)(1+\cos \theta)^{2}$, the asymmetry provides a clean probe of $\Delta \bar{u}\left(x_{1}\right) / \bar{u}\left(x_{1}\right)$ at medium values of $x_{1}$. By similar reasoning, at forward rapidity $\eta_{\text {lept }} \gg 0$ the second term in Eq. (10) dominates, giving access to $-\Delta d\left(x_{1}\right) / d\left(x_{1}\right)$ at relatively high $x_{1}$.

For $W^{+}$production, one has the following structure of the spin-dependent cross section:

$$
\Delta \sigma \propto \Delta \bar{d}\left(x_{1}\right) \otimes u\left(x_{2}\right)(1+\cos \theta)^{2}-\Delta u\left(x_{1}\right) \otimes \bar{d}\left(x_{2}\right)(1-\cos \theta)^{2} .
$$

Here the distinction of the two contributions by considering large negative or positive lepton rapidities is less clear-cut than in the case of $W^{-}$. For example, at negative $\eta_{\text {lept }}$ the partonic combination $\bar{d}\left(x_{1}\right) u\left(x_{2}\right)$ will dominate, but at the same time $\theta \gg \pi / 2$ so that the angular factor $(1+\cos \theta)^{2}$ is small. Likewise, at positive $\eta_{\text {lept }}$ the dominant partonic combination $\Delta u\left(x_{1}\right) \bar{d}\left(x_{2}\right)$ is suppressed by the angular factor. So both terms in Eq. (11) will compete essentially for all $\eta_{\text {lept }}$ of interest. Our global analysis technique is of course suited for extracting information on the polarized PDFs even if there is no single dominant partonic subprocess. The NLO corrections to the single-inclusive lepton cross sections have recently been presented in [37] in a way tailored to use in the global analysis framework.

Figure 5 shows the first published data from RHIC for $A_{L}$ in $W^{ \pm}$production [38, 39]. For now, the statistical uncertainties are still large. However, already now a large negative asymmetry is seen for the case of $W^{+}$production, resulting primarily from the positive up-quark polarization in the proton (see Eq. (11)). Clearly, there is a large potential in future $W$-measurements at RHIC.

\subsection{New constraints on $\Delta g$}

The STAR and PHENIX experiments at RHIC have recently presented new preliminary data from the 2009 run for the double-helicity spin asymmetry $A_{L L}$ for jet and neutral-pion production, respectively [40, 41]. The results are shown in Figs. 6 and 7. One can see that the experimental uncertainties 

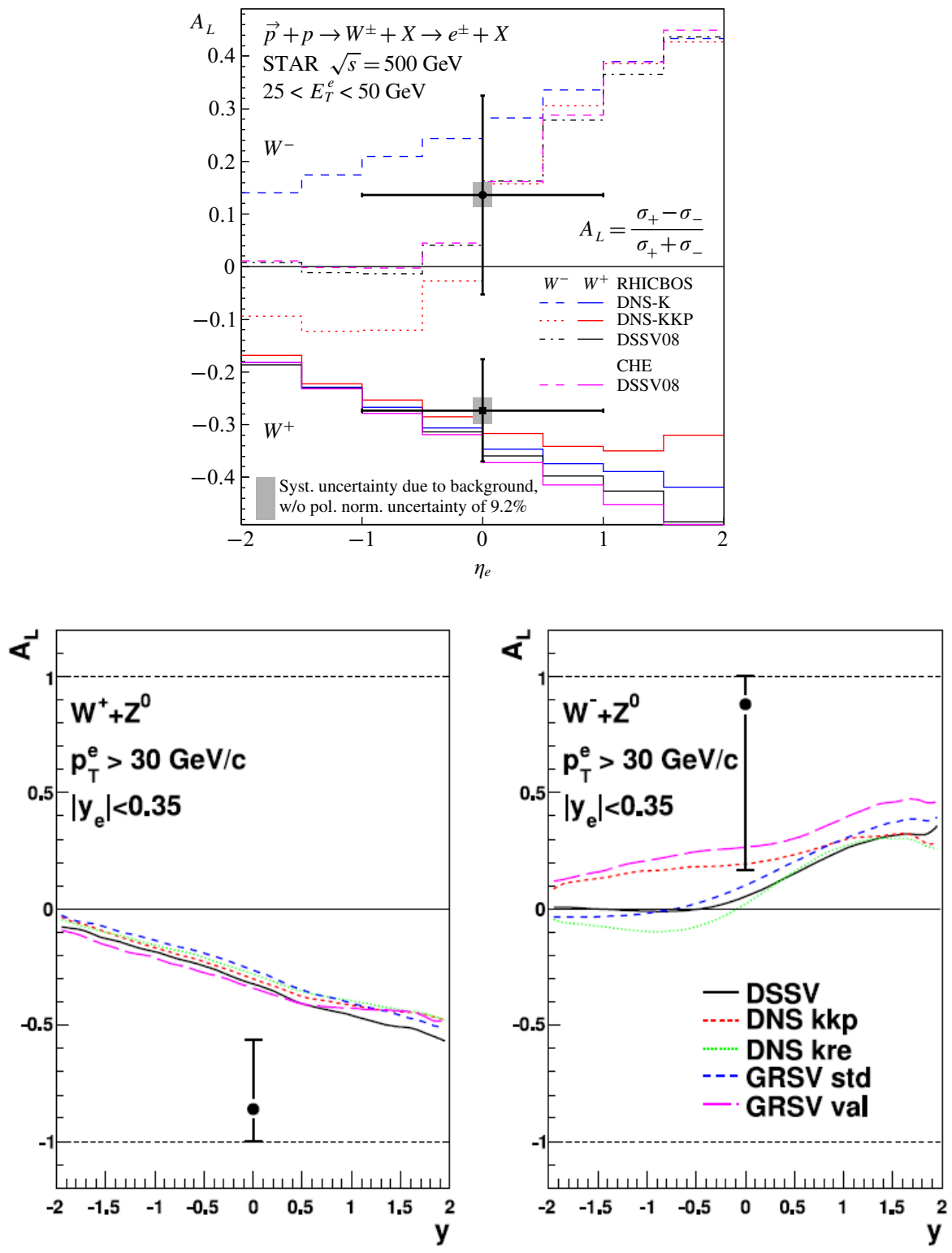

Figure 5: Published STAR [38] (top) and PHENIX [39] (bottom) data for the single-helicity asymmetry $A_{L}$ in $W^{ \pm}$production at RHIC.

are very significantly reduced as compared to those in the previous run-6 data sets [42, 43]. An interesting feature of the new preliminary STAR data is that they lie consistently above the result for the best-fit DSSV distribution for jet transverse momenta below $25 \mathrm{GeV}$ or so. They do remain well below the old GRSV-"standard" result of [14], on the other hand. This suggests that the spin-dependent gluon distribution may be somewhat different from zero in the $x$-range where it is constrained by the RHIC data. The thick (magenta) solid line in the figure shows $A_{L L}$ obtained for a special set of parton distributions within the DSSV analysis. For this set the truncated moment of $\Delta g$ over the region $0.001 \leq x \leq 1$ was varied, allowing the total $\chi^{2}$ to change by $2 \%$. Evidently, this set of parton distributions describes the STAR data rather well. The truncated moment of $\Delta g$ in this set over the $x$-range accessed at RHIC is

$$
\int_{0.05}^{0.2} d x \Delta g\left(x, Q^{2}=10 \mathrm{GeV}^{2}\right)=0.13
$$




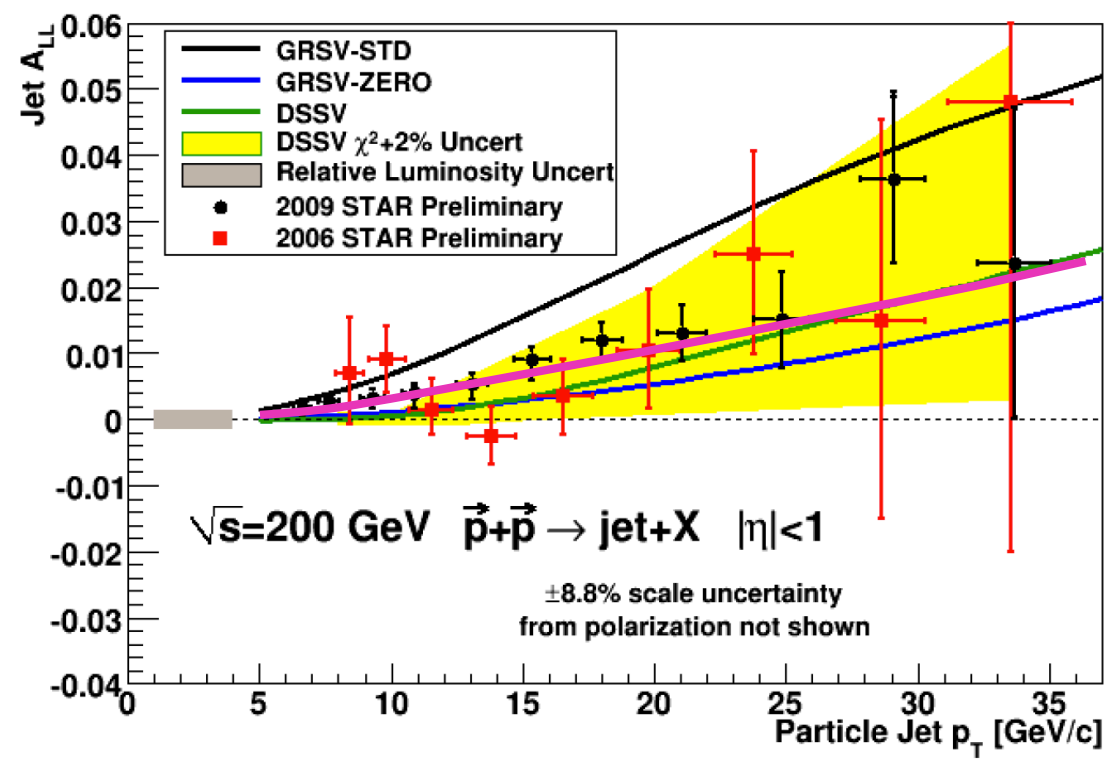

Figure 6: Preliminary STAR run-9 data [40] for the double-helicity asymmetry $A_{L L}$ for single-inclusive jet production, as a function of jet transverse momentum for $|\eta|<1$. The preliminary run- 6 data are shown as well. The theoretical curves are as described in the caption. The additional solid magenta line gives the result for a special DSSV set of polarized parton distributions, for which the truncated moment of $\Delta g$ over the region $0.001 \leq x \leq 1$ was varied allowing the total $\chi^{2}$ of the fit to change by $2 \%$.

which is just within the range

$$
\int_{0.05}^{0.2} d x \Delta g\left(x, Q^{2}=10 \mathrm{GeV}^{2}\right)=0.005_{-0.164}^{+0.129}
$$

quoted as more conservative uncertainty $\left(\Delta \chi^{2} / \chi^{2}=2 \%\right)$ in [13. Despite the fact that this really is only an illustration that cannot replace a proper re-analysis of the data, it does appears that, for the first time, there are indications of non-vanishing gluon polarization in the nucleon. Figure 7 shows the comparison to the new preliminary PHENIX $\pi^{0}$ data [41]. Here the values of $A_{L L}$ are much smaller, which is mostly due to the fact that lower values of $x$ are probed at the transverse momenta relevant in the PHENIX measurements. One can see that the data are well described by both the DSSV set and the special set of polarized parton distributions used in Fig. 6.

\section{The future: Electron Ion Collider (EIC)}

An Electron Ion Collider is currently being considered in the U.S. as a new frontier facility to explore strong-interaction phenomena [31]. One of its key features would be the availability of high-energy, high-luminosity polarized $e p$ collisions to probe nucleon spin structure. This would also allow precision extractions of $\Delta g$, in particular from scaling violations of the proton's spin-dependent structure function $g_{1}$. Figure 8 shows the results of a recent dedicated phenomenological study [44]. "Pseudo" EIC-data were generated for collisions of $5 \mathrm{GeV}$ electrons with 50,100, 250, and $325 \mathrm{GeV}$ protons and were added to the DSSV global analysis code. The statistical precision of the data sets for $100-325 \mathrm{GeV}$ protons was taken to correspond to about two months of running at the anticipated luminosities for eRHIC with an assumed operations efficiency of $50 \%$. For $5 \times 50 \mathrm{GeV}$ an integrated luminosity of $5 \mathrm{fb}^{-1} \mathrm{was}^{-}$ 


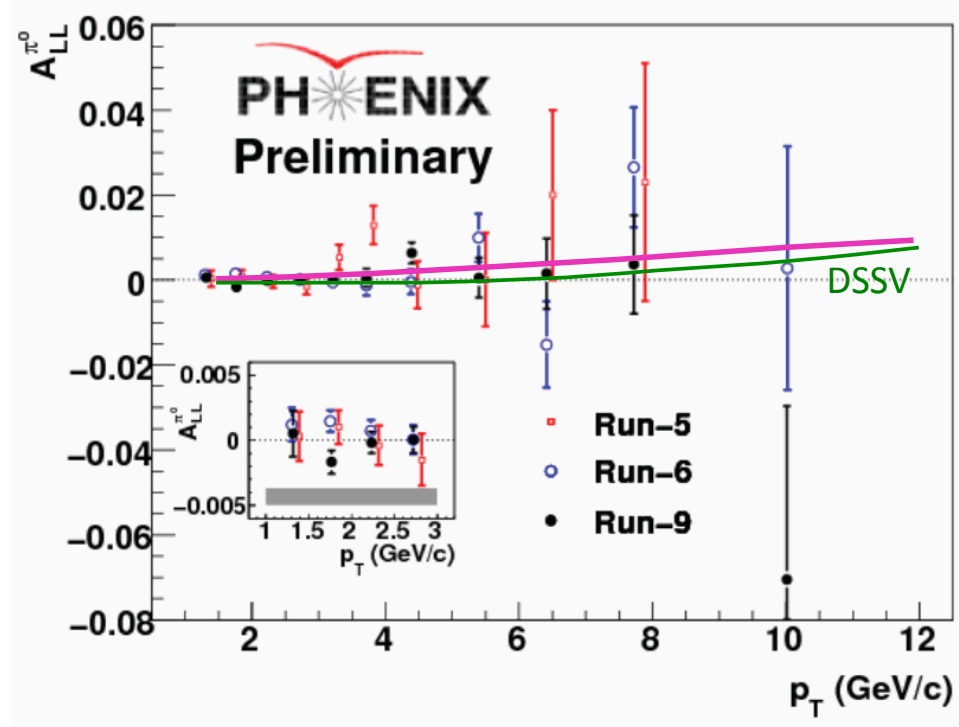

Figure 7: As in Fig. 6, but for the preliminary PHENIX run-9 data [41.

assumed. The projected uncertainties were used to randomize the pseudo-data by one sigma around their central values determined by the DSSV set of PDFs. With these projected EIC data with their estimated uncertainties, a re-fit of the DSSV polarized parton distribution functions was performed. The results are shown in Fig. 8 . As one can see, with EIC data it should be possible to map the currently completely undetermined shape of $\Delta g$ for $10^{-4} \lesssim x \lesssim 0.01$ to an accuracy of about $\pm 10 \%$ or better.

\section{Acknowledgments}

This work was supported in part by the U.S. Department of Energy (contract number DE-AC0298CH10886), and by CONICET, ANPCyT and UBACyT.

\section{References}

[1] For a recent review, see: M. Burkardt, C. A. Miller and W. D. Nowak, Rept. Prog. Phys. 73 (2010) 016201.

[2] R. L. Jaffe and A. Manohar, Nucl. Phys. B 337 (1990) 509; X. -D. Ji, Phys. Rev. Lett. 78 (1997) 610; S. Bashinsky and R. L. Jaffe, Nucl. Phys. B 536 (1998) 303; M. Wakamatsu, Phys. Rev. D 81 (2010) 114010; Phys. Rev. D 83 (2011) 014012. E. Leader, Phys. Rev. D 83 (2011) 096012.

[3] G. Altarelli and G. Parisi, Nucl. Phys. B 126 (1977) 298; M. A. Ahmed and G. G. Ross, Nucl. Phys. B 111 (1976) 441.

[4] R. Mertig and W. L. van Neerven, Z. Phys. C 70 (1996) 637; W. Vogelsang, Phys. Rev. D 54 (1996) 2023; Nucl. Phys. B 475 (1996) 47.

[5] A. Vogt, S. Moch, M. Rogal and J. A. M. Vermaseren, Nucl. Phys. Proc. Suppl. 183 (2008) 155.

[6] A. V. Manohar, Phys. Rev. Lett. 66 (1991) 289.

[7] G. Bunce, N. Saito, J. Soffer and W. Vogelsang, Ann. Rev. Nucl. Part. Sci. 50 (2000) 525. 


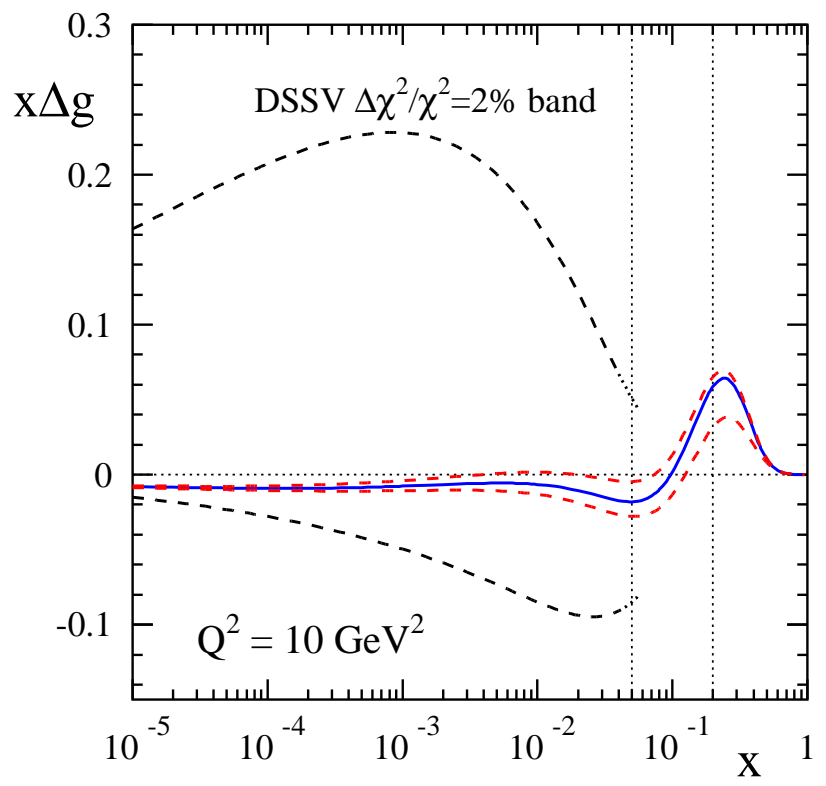

Figure 8: Uncertainty bands for $x \Delta g$ referring to $\Delta \chi^{2} / \chi^{2}=2 \%$ with and without including the generated EIC pseudo-data in the fit. The dashed lines correspond to the yellow band for $\Delta g$ shown in Fig. 2.

[8] G. Bunce et al., http://spin.riken.bnl.gov/rsc/report/spinplan_2008/spinplan08.pdf.

[9] J. C. Collins, Nucl. Phys. B 394 (1993) 169.

[10] See, for example: L. E. Gordon, W. Vogelsang, Phys. Rev. D 48 (1993) 3136; Phys. Rev. D 50 (1994) 1901; D. de Florian, Phys. Rev. D 67 (2003) 054004; B. Jäger, A. Schäfer, M. Stratmann and W. Vogelsang, Phys. Rev. D 67 (2003) 054005; B. Jäger, M. Stratmann, W. Vogelsang, Phys. Rev. D 70 (2004) 034010; I. Bojak and M. Stratmann, Phys. Lett. B 433 (1998) 411; Nucl. Phys. B 540 (1999) 345; Phys. Rev. D 67 (2003) 034010; B. Jäger, M. Stratmann and W. Vogelsang, Phys. Rev. D 68 (2003) 114018; C. Hendlmeier, M. Stratmann, A. Schäfer, Eur. Phys. J. C 48 (2006) 135; Eur. Phys. J. C 55 (2008) 597.

[11] M. J. Savage and J. Walden, Phys. Rev. D 55 (1997) 5376.

[12] G. Bali et al. [QCDSF Collaboration], PoSLAT 2009 (2009) 149; G. Bali, these proceedings.

[13] D. de Florian, R. Sassot, M. Stratmann, W. Vogelsang, Phys. Rev. Lett. 101 (2008) 072001; Phys. Rev. D80 (2009) 034030.

[14] M. Glück, E. Reya, M. Stratmann and W. Vogelsang, Phys. Rev. D 63 (2001) 094005; Phys. Rev. D 53 (1996) 4775.

[15] D. de Florian, G. A. Navarro and R. Sassot, Phys. Rev. D 71 (2005) 094018.

[16] E. Leader, A. V. Sidorov, D. B. Stamenov, arXiv:1012.5033 [hep-ph].

[17] J. Blümlein, H. Böttcher, Nucl. Phys. B 841 (2010) 205.

[18] C. Bourrely, J. Soffer and F. Buccella, Eur. Phys. J. C 23 (2002) 487.

[19] D. de Florian, R. Sassot and M. Stratmann, Phys. Rev. D 75 (2007) 114010; Phys. Rev. D 76 (2007) 074033.

[20] D. Diakonov et al., Nucl. Phys. B 480 (1996) 341; Phys. Rev. D 56 (1997) 4069; M. Wakamatsu and T. Kubota, Phys. Rev. D 60 (1999) 034020; B. Dressler, K. Goeke, M. V. Polyakov, P. Schweitzer, M. Strikman and C. Weiss, Eur. Phys. J. C 18 (2001) 719; S. Kumano, Phys. Rept. 303 (1998) 183 
M. Glück and E. Reya, Mod. Phys. Lett. A 15 (2000) 883; F. G. Cao and A. I. Signal, Eur. Phys. J. C 21 (2001) 105; R. S. Bhalerao, Phys. Rev. C 63 (2001) 025208; R. J. Fries, A. Schäfer and C. Weiss, Eur. Phys. J. A17 (2003) 509.

[21] For an overview of the latest results, see: J. Pretz [COMPASS Collaboration], arXiv:1109.2446 [hep-ex].

[22] A. Airapetian et al. [HERMES Collaboration], JHEP 1008 (2010) 130, and references therein.

[23] A. Morreale [COMPASS Collaboration], talk presented at "Hadron Structure 11", Tatranska Strba, Slovakia, June 2011 (http://wwwcompass.cern.ch/compass/publications/talks/); C. Hoeppner [COMPASS Collaboration], TU Munich thesis 2011.

[24] D. de Florian, M. Pfeuffer, A. Schäfer, W. Vogelsang, in preparation.

[25] M. G. Alekseev et al. [COMPASS Collaboration], Phys. Lett. B 690 (2010) 466.

[26] M. G. Alekseev et al. [COMPASS Collaboration], Phys. Lett. B 680 (2009) 217.

[27] M. G. Alekseev et al. [COMPASS Collaboration], Phys. Lett. B 693 (2010) 227.

[28] K. Ackerstaff et al. [HERMES Collaboration], Phys. Lett. B 464 (1999) 123; A. Airapetian et al. [HERMES Collaboration], Phys. Rev. D 71 (2005) 012003; A. Airapetian et al. [HERMES Collaboration], Phys. Lett. B 666 (2008) 446.

[29] M. Stratmann, talk presented at the "XIX International Workshop on Deep-Inelastic Scattering and Related Subjects (DIS 2011)", April 2011, Newport News, VA USA. Proceedings contribution: D. de Florian, R. Sassot, M. Stratmann and W. Vogelsang, arXiv:1108.3955 [hep-ph].

[30] E. Aschenauer and M. Stratmann, in Ref. [31].

[31] D. Boer, M. Diehl, R. Milner, R. Venugopalan, W. Vogelsang, D. Kaplan, H. Montgomery and S. Vigdor et al., arXiv:1108.1713 [nucl-th].

[32] For review, see: S. Albino, F. Anulli, F. Arleo, D. Z. Besson, W. K. Brooks, B. Buschbeck, M. Cacciari and E. Christova et al., arXiv:0804.2021 [hep-ph].

[33] C. Bourrely, J. Soffer, and E. Leader, Phys. Rep. 59 (1980) 95; N. S. Craigie, K. Hidaka, M. Jacob, and F. M. Renard, Phys. Rep. 99 (1983) 69; P. Chiappetta and J. Soffer, Phys. Lett. B 152 (1985) 126; C. Bourrely and J. Soffer, Phys. Lett. B 314 (1993) 132; Nucl. Phys. B 423 (1994) 329; Nucl. Phys. B 445 (1995) 341.

[34] B. Kamal, Phys. Rev. D 57 (1998) 6663; T. Gehrmann, Nucl. Phys. B 534 (1998) 21.

[35] P. M. Nadolsky and C. P. Yuan, Nucl. Phys. B 666 (2003) 31.

[36] A. Mukherjee and W. Vogelsang, Phys. Rev. D 73 (2006) 074005.

[37] D. de Florian and W. Vogelsang, Phys. Rev. D 81 (2010) 094020.

[38] M. M. Aggarwal et al. [STAR Collaboration], Phys. Rev. Lett. 106 (2011) 062002.

[39] A. Adare et al. [PHENIX Collaboration], Phys. Rev. Lett. 106 (2011) 062001.

[40] P. Djawotho [STAR Collaboration], talk presented at the "XIX International Workshop on Deep-Inelastic Scattering and Related Subjects (DIS 2011)", April 2011, Newport News, VA USA (https://wiki.bnl.gov/conferences/index.php/Spin_Physics), J. Phys. Conf. Ser. 295 (2011) 012061.

[41] A. Datta [PHENIX Collaboration], talk presented at the "XIX International Workshop on DeepInelastic Scattering and Related Subjects (DIS 2011)", April 2011, Newport News, VA USA (https://wiki.bnl.gov/conferences/index.php/Spin_Physics).

[42] B. Surrow [STAR Collaboration], PoSDIS 2010 (2010) 249.

[43] A. Adare et al. [PHENIX Collaboration], Phys. Rev. Lett. 103 (2009) 012003.

[44] R. Sassot and M. Stratmann, in Ref. [31]. 